\title{
O DESENVOLVIMENTO SUSTENTÁVEL NA ENGENHARIA CIVIL 2
}

Henrique Ajuz Holzmann

João Dallamuta

(Organizadores)

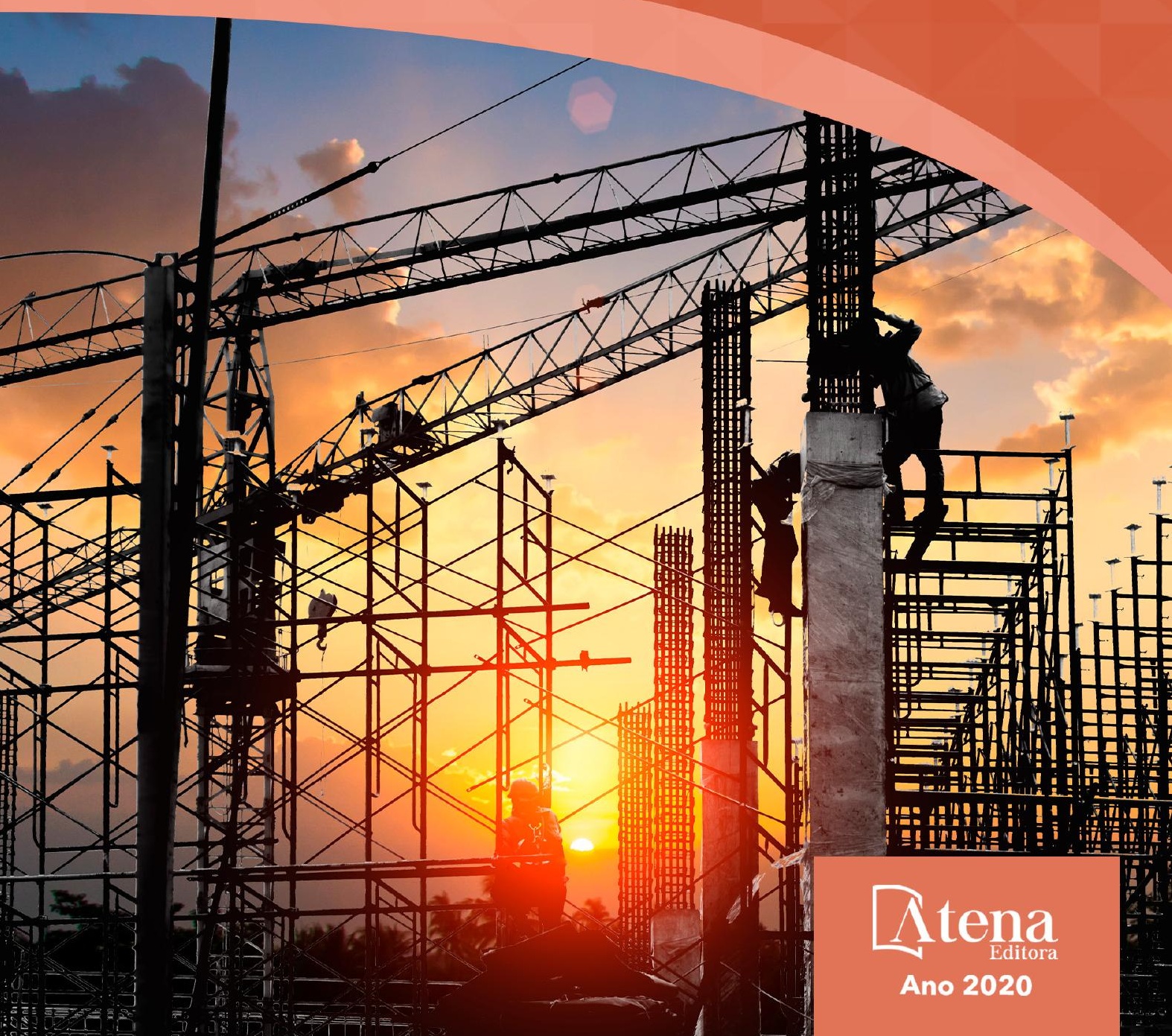




\section{O DESENVOLVIMENTO SUSTENTÁVEL NA ENGENHARIA CIVIL 2}

Henrique Ajuz Holzmann

João Dallamuta

(Organizadores)

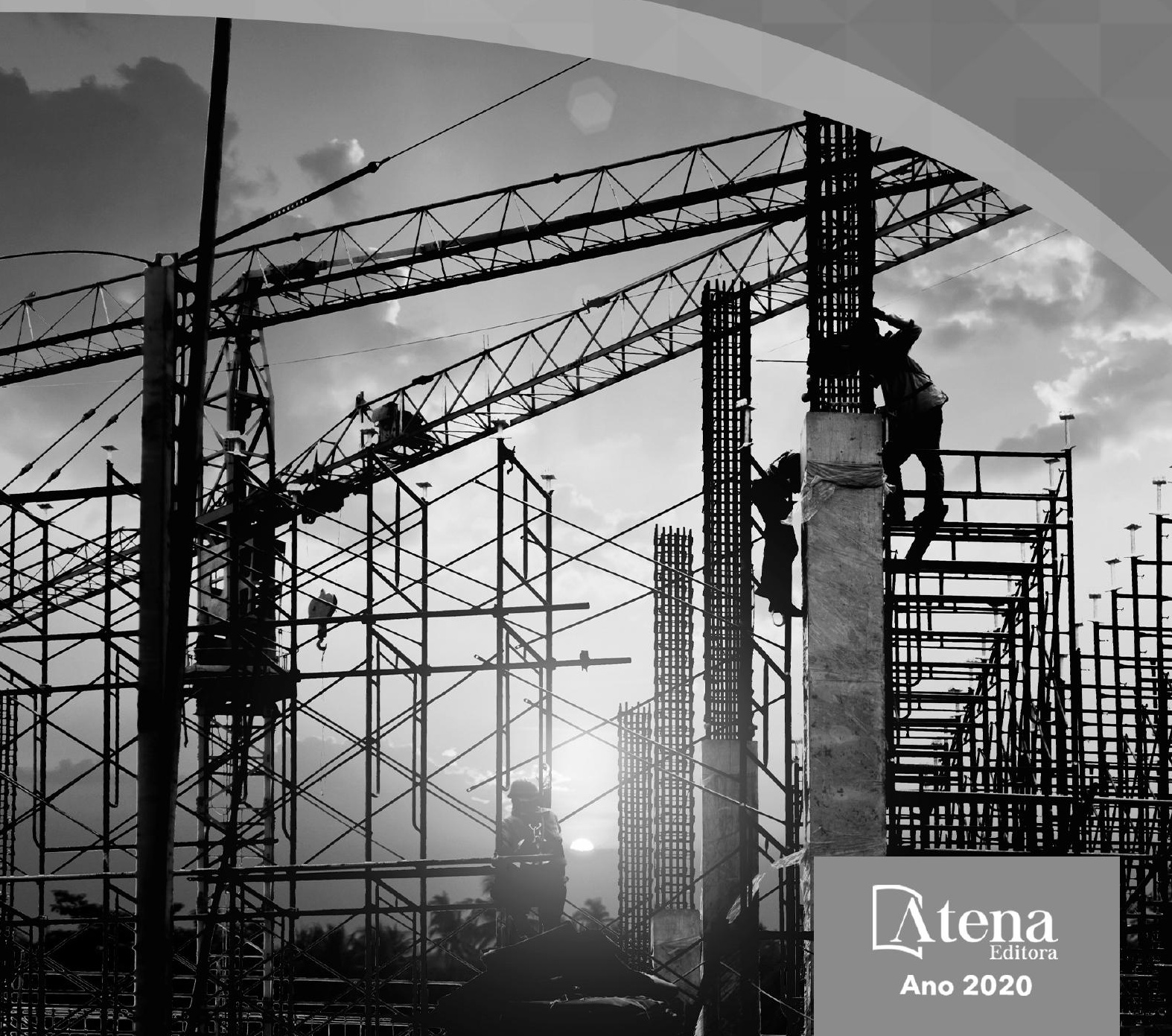




\section{Editora Chefe}

Prof $^{\mathrm{a}}$ Dr $^{\mathrm{a}}$ Antonella Carvalho de Oliveira

Assistentes Editoriais

Natalia Oliveira

Bruno Oliveira

Flávia Roberta Barão

Bibliotecária

Janaina Ramos

Projeto Gráfico e Diagramação

Natália Sandrini de Azevedo

Camila Alves de Cremo

Luiza Alves Batista

Maria Alice Pinheiro

Imagens da Capa

Shutterstock

Edição de Arte

Luiza Alves Batista

Revisão

Os Autores

\section{0 by Atena Editora}

Copyright (C) Atena Editora

Copyright do Texto (c) 2020 Os autores

Copyright da Edição (c) 2020 Atena Editora

Direitos para esta edição cedidos à Atena

Editora pelos autores.

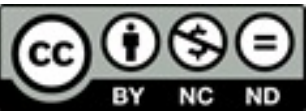

Todo o conteúdo deste livro está licenciado sob uma Licença de Atribuição Creative Commons. Atribuição-Não-ComercialNãoDerivativos 4.0 Internacional (CC BY-NC-ND 4.0).

O conteúdo dos artigos e seus dados em sua forma, correção e confiabilidade são de responsabilidade exclusiva dos autores, inclusive não representam necessariamente a posição oficial da Atena Editora. Permitido o download da obra e o compartilhamento desde que sejam atribuídos créditos aos autores, mas sem a possibilidade de alterá-la de nenhuma forma ou utilizá-la para fins comerciais.

Todos os manuscritos foram previamente submetidos à avaliação cega pelos pares, membros do Conselho Editorial desta Editora, tendo sido aprovados para a publicação.

A Atena Editora é comprometida em garantir a integridade editorial em todas as etapas do processo de publicação. Situações suspeitas de má conduta científica serão investigadas sob o mais alto padrão de rigor acadêmico e ético.

\section{Conselho Editorial}

Ciências Humanas e Sociais Aplicadas

Prof. Dr. Alexandre Jose Schumacher - Instituto Federal de Educação, Ciência e Tecnologia do Paraná

Prof. Dr. Américo Junior Nunes da Silva - Universidade do Estado da Bahia

Prof. Dr. Antonio Carlos Frasson - Universidade Tecnológica Federal do Paraná

Prof. Dr. Antonio Gasparetto Júnior - Instituto Federal do Sudeste de Minas Gerais

Prof. Dr. Antonio Isidro-Filho - Universidade de Brasília

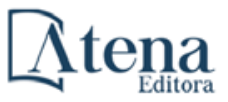


Prof. Dr. Carlos Antonio de Souza Moraes - Universidade Federal Fluminense

Prof $^{a}$ Dr $^{\text {a }}$ Cristina Gaio - Universidade de Lisboa

Prof. Dr. Daniel Richard Sant'Ana - Universidade de Brasília

Prof. Dr. Deyvison de Lima Oliveira - Universidade Federal de Rondônia

Prof $^{a}$ Dr $^{a}$ Dilma Antunes Silva - Universidade Federal de São Paulo

Prof. Dr. Edvaldo Antunes de Farias - Universidade Estácio de Sá

Prof. Dr. Elson Ferreira Costa - Universidade do Estado do Pará

Prof. Dr. Eloi Martins Senhora - Universidade Federal de Roraima

Prof. Dr. Gustavo Henrique Cepolini Ferreira - Universidade Estadual de Montes Claros

Prof $^{a} \mathrm{Dr}^{\mathrm{a}}$ Ivone Goulart Lopes - Istituto Internazionele delle Figlie de Maria Ausiliatrice

Prof. Dr. Jadson Correia de Oliveira - Universidade Católica do Salvador

Prof. Dr. Julio Candido de Meirelles Junior - Universidade Federal Fluminense

Prof $^{a} \mathrm{Dr}^{\mathrm{a}}$ Lina Maria Gonçalves - Universidade Federal do Tocantins

Prof. Dr. Luis Ricardo Fernandes da Costa - Universidade Estadual de Montes Claros

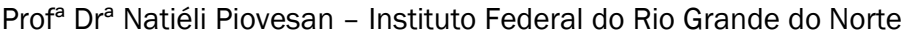

Prof. Dr. Marcelo Pereira da Silva - Pontifícia Universidade Católica de Campinas

Prof $^{a}$ Dr $^{a}$ Maria Luzia da Silva Santana - Universidade Federal de Mato Grosso do Sul

Prof $^{a}$ Dr $^{a}$ Paola Andressa Scortegagna - Universidade Estadual de Ponta Grossa

Prof $^{a}$ Dr $^{a}$ Rita de Cássia da Silva Oliveira - Universidade Estadual de Ponta Grossa

Prof. Dr. Rui Maia Diamantino - Universidade Salvador

Prof. Dr. Urandi João Rodrigues Junior - Universidade Federal do Oeste do Pará

Prof $^{a}$ Dr $^{a}$ Vanessa Bordin Viera - Universidade Federal de Campina Grande

Prof. Dr. William Cleber Domingues Silva - Universidade Federal Rural do Rio de Janeiro

Prof. Dr. Willian Douglas Guilherme - Universidade Federal do Tocantins

\section{Ciências Agrárias e Multidisciplinar}

Prof. Dr. Alexandre Igor Azevedo Pereira - Instituto Federal Goiano

Prof $^{\mathrm{a}} \mathrm{Dr}^{\mathrm{a}}$ Carla Cristina Bauermann Brasil - Universidade Federal de Santa Maria

Prof. Dr. Antonio Pasqualetto - Pontifícia Universidade Católica de Goiás

Prof. Dr. Cleberton Correia Santos - Universidade Federal da Grande Dourados

Prof $^{a}$ Dr $^{a}$ Daiane Garabeli Trojan - Universidade Norte do Paraná

Prof ${ }^{a}$ Dr $^{a}$ Diocléa Almeida Seabra Silva - Universidade Federal Rural da Amazônia

Prof. Dr. Écio Souza Diniz - Universidade Federal de Viçosa

Prof. Dr. Fábio Steiner - Universidade Estadual de Mato Grosso do Sul

Prof. Dr. Fágner Cavalcante Patrocínio dos Santos - Universidade Federal do Ceará

Prof ${ }^{a}$ Dr $^{a}$ Girlene Santos de Souza - Universidade Federal do Recôncavo da Bahia

Prof. Dr. Jael Soares Batista - Universidade Federal Rural do Semi-Árido

Prof. Dr. Júlio César Ribeiro - Universidade Federal Rural do Rio de Janeiro

Prof $^{a}$ Dr $^{a}$ Lina Raquel Santos Araújo - Universidade Estadual do Ceará

Prof. Dr. Pedro Manuel Villa - Universidade Federal de Viçosa

Prof $^{a}$ Dr $^{a}$ Raissa Rachel Salustriano da Silva Matos - Universidade Federal do Maranhão

Prof. Dr. Ronilson Freitas de Souza - Universidade do Estado do Pará

Prof $^{a}$ Dr $^{a}$ Talita de Santos Matos - Universidade Federal Rural do Rio de Janeiro

Prof. Dr. Tiago da Silva Teófilo - Universidade Federal Rural do Semi-Árido

Prof. Dr. Valdemar Antonio Paffaro Junior - Universidade Federal de Alfenas

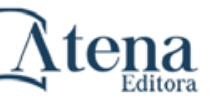

Ano 2020 


\section{Ciências Biológicas e da Saúde}

Prof. Dr. André Ribeiro da Silva - Universidade de Brasília

Prof $^{a}$ Dr $^{a}$ Anelise Levay Murari - Universidade Federal de Pelotas

Prof. Dr. Benedito Rodrigues da Silva Neto - Universidade Federal de Goiás

Prof $^{a}$ Dr $^{a}$ Débora Luana Ribeiro Pessoa - Universidade Federal do Maranhão

Prof. Dr. Douglas Siqueira de Almeida Chaves -Universidade Federal Rural do Rio de Janeiro

Prof. Dr. Edson da Silva - Universidade Federal dos Vales do Jequitinhonha e Mucuri

Prof $^{a}$ Dr $^{a}$ Eleuza Rodrigues Machado - Faculdade Anhanguera de Brasília

Prof $^{\mathrm{a}} \mathrm{Dr}^{\mathrm{a}}$ Elane Schwinden Prudêncio - Universidade Federal de Santa Catarina

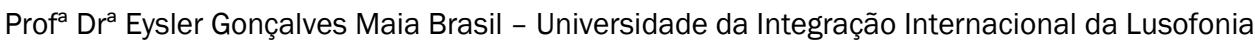
Afro-Brasileira

Prof. Dr. Ferlando Lima Santos - Universidade Federal do Recôncavo da Bahia

Prof $^{a}$ Dr $^{\text {a }}$ Gabriela Vieira do Amaral - Universidade de Vassouras

Prof. Dr. Gianfábio Pimentel Franco - Universidade Federal de Santa Maria

Prof. Dr. Helio Franklin Rodrigues de Almeida - Universidade Federal de Rondônia

Prof $^{a}$ Dr $^{a}$ lara Lúcia Tescarollo - Universidade São Francisco

Prof. Dr. Igor Luiz Vieira de Lima Santos - Universidade Federal de Campina Grande

Prof. Dr. Jefferson Thiago Souza - Universidade Estadual do Ceará

Prof. Dr. Jesus Rodrigues Lemos - Universidade Federal do Piauí

Prof. Dr. Jônatas de França Barros - Universidade Federal do Rio Grande do Norte

Prof. Dr. José Max Barbosa de Oliveira Junior - Universidade Federal do Oeste do Pará

Prof. Dr. Luís Paulo Souza e Souza - Universidade Federal do Amazonas

Prof ${ }^{a}$ Dr $^{a}$ Magnólia de Araújo Campos - Universidade Federal de Campina Grande

Prof. Dr. Marcus Fernando da Silva Praxedes - Universidade Federal do Recôncavo da Bahia

Prof $^{\mathrm{a}} \mathrm{Dr}^{\mathrm{a}}$ Maria Tatiane Gonçalves Sá - Universidade do Estado do Pará

Prof $^{\mathrm{a}} \mathrm{Dr}^{\mathrm{a}}$ Mylena Andréa Oliveira Torres - Universidade Ceuma

Prof $^{a}$ Dr $^{a}$ Natiéli Piovesan - Instituto Federacl do Rio Grande do Norte

Prof. Dr. Paulo Inada - Universidade Estadual de Maringá

Prof. Dr. Rafael Henrique Silva - Hospital Universitário da Universidade Federal da Grande Dourados

Prof $^{a}$ Dr $^{a}$ Regiane Luz Carvalho - Centro Universitário das Faculdades Associadas de Ensino

Prof $^{\mathrm{a}} \mathrm{Dr}^{\mathrm{a}}$ Renata Mendes de Freitas - Universidade Federal de Juiz de Fora

Prof $^{a}$ Dr $^{a}$ Vanessa Lima Gonçalves - Universidade Estadual de Ponta Grossa

Prof $^{a}$ Dr $^{a}$ Vanessa Bordin Viera - Universidade Federal de Campina Grande

\section{Ciências Exatas e da Terra e Engenharias}

Prof. Dr. Adélio Alcino Sampaio Castro Machado - Universidade do Porto

Prof. Dr. Carlos Eduardo Sanches de Andrade - Universidade Federal de Goiás

Prof ${ }^{a}$ Dr $^{a}$ Carmen Lúcia Voigt - Universidade Norte do Paraná

Prof. Dr. Douglas Gonçalves da Silva - Universidade Estadual do Sudoeste da Bahia

Prof. Dr. Eloi Rufato Junior - Universidade Tecnológica Federal do Paraná

Prof $^{a}$ Dr $^{a}$ Érica de Melo Azevedo - Instituto Federal do Rio de Janeiro

Prof. Dr. Fabrício Menezes Ramos - Instituto Federal do Pará

Prof $^{a}$ Dra. Jéssica Verger Nardeli - Universidade Estadual Paulista Júlio de Mesquita Filho

Prof. Dr. Juliano Carlo Rufino de Freitas - Universidade Federal de Campina Grande

Prof $^{a}$ Dr $^{a}$ Luciana do Nascimento Mendes - Instituto Federal de Educação, Ciência e Tecnologia

do Rio Grande do Norte

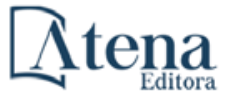


Prof. Dr. Marcelo Marques - Universidade Estadual de Maringá

Prof $^{a} \operatorname{Dr}^{\mathrm{a}}$ Neiva Maria de Almeida - Universidade Federal da Paraíba

Prof $^{a}$ Dr $^{a}$ Natiéli Piovesan - Instituto Federal do Rio Grande do Norte

Prof ${ }^{a}$ Dr $^{\text {a }}$ Priscila Tessmer Scaglioni - Universidade Federal de Pelotas

Prof. Dr. Takeshy Tachizawa - Faculdade de Campo Limpo Paulista

\section{Linguística, Letras e Artes}

Prof $^{a}$ Dr $^{a}$ Adriana Demite Stephani - Universidade Federal do Tocantins

Prof $^{a}$ Dr $^{a}$ Angeli Rose do Nascimento - Universidade Federal do Estado do Rio de Janeiro

Prof $^{a}$ Dra $^{\text {a }}$ Carolina Fernandes da Silva Mandaji - Universidade Tecnológica Federal do Paraná

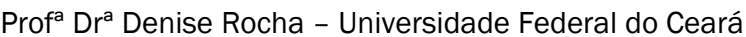

Prof. Dr. Fabiano Tadeu Grazioli - Universidade Regional Integrada do Alto Uruguai e das Missões

Prof. Dr. Gilmei Fleck - Universidade Estadual do Oeste do Paraná

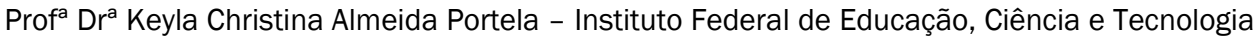
do Paraná

Prof $^{a}$ Dra $^{a}$ Miranilde Oliveira Neves - Instituto de Educação, Ciência e Tecnologia do Pará

Prof $^{a}$ Dr $^{\text {a }}$ Sandra Regina Gardacho Pietrobon - Universidade Estadual do Centro-Oeste

Prof $^{a}$ Dr $^{a}$ Sheila Marta Carregosa Rocha - Universidade do Estado da Bahia

\section{Conselho Técnico Científico}

Prof. Me. Abrãao Carvalho Nogueira - Universidade Federal do Espírito Santo

Prof. Me. Adalberto Zorzo - Centro Estadual de Educação Tecnológica Paula Souza

Prof. Me. Adalto Moreira Braz - Universidade Federal de Goiás

Prof. Dr. Adaylson Wagner Sousa de Vasconcelos - Ordem dos Advogados do Brasil/Seccional Paraíba

Prof. Dr. Adilson Tadeu Basquerote Silva - Universidade para o Desenvolvimento do Alto Vale do Itajaí

Prof. Me. Alexsandro Teixeira Ribeiro - Centro Universitário Internacional

Prof. Me. André Flávio Gonçalves Silva - Universidade Federal do Maranhão

Prof ${ }^{a}$ Ma. Andréa Cristina Marques de Araújo - Universidade Fernando Pessoa

Prof $^{a}$ Dr $^{\text {a }}$ Andreza Lopes - Instituto de Pesquisa e Desenvolvimento Acadêmico

Prof $^{a}$ Dr $^{a}$ Andrezza Miguel da Silva - Faculdade da Amazônia

Profa Ma. Anelisa Mota Gregoleti - Universidade Estadual de Maringá

Prof ${ }^{a}$ Ma. Anne Karynne da Silva Barbosa - Universidade Federal do Maranhão

Prof. Dr. Antonio Hot Pereira de Faria - Polícia Militar de Minas Gerais

Prof. Me. Armando Dias Duarte - Universidade Federal de Pernambuco

Prof $^{a}$ Ma. Bianca Camargo Martins - UniCesumar

Prof $^{a}$ Ma. Carolina Shimomura Nanya - Universidade Federal de São Carlos

Prof. Me. Carlos Antônio dos Santos - Universidade Federal Rural do Rio de Janeiro

Prof. Ma. Cláudia de Araújo Marques - Faculdade de Música do Espírito Santo

Prof $^{a}$ Dr $^{a}$ Cláudia Taís Siqueira Cagliari - Centro Universitário Dinâmica das Cataratas

Prof. Me. Clécio Danilo Dias da Silva - Universidade Federal do Rio Grande do Norte

Prof. Me. Daniel da Silva Miranda - Universidade Federal do Pará

Prof $^{a}$ Ma. Daniela da Silva Rodrigues - Universidade de Brasília

Prof $^{\mathrm{a}}$ Ma. Daniela Remião de Macedo - Universidade de Lisboa

Prof $^{a}$ Ma. Dayane de Melo Barros - Universidade Federal de Pernambuco

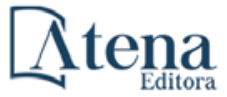


Prof. Me. Douglas Santos Mezacas - Universidade Estadual de Goiás

Prof. Me. Edevaldo de Castro Monteiro - Embrapa Agrobiologia

Prof. Me. Eduardo Gomes de Oliveira - Faculdades Unificadas Doctum de Cataguases

Prof. Me. Eduardo Henrique Ferreira - Faculdade Pitágoras de Londrina

Prof. Dr. Edwaldo Costa - Marinha do Brasil

Prof. Me. Eliel Constantino da Silva - Universidade Estadual Paulista Júlio de Mesquita

Prof. Me. Ernane Rosa Martins - Instituto Federal de Educação, Ciência e Tecnologia de Goiás

Prof. Me. Euvaldo de Sousa Costa Junior - Prefeitura Municipal de São João do Piauí

Prof $^{a}$ Ma. Fabiana Coelho Couto Rocha Corrêa - Centro Universitário Estácio Juiz de Fora

Prof. Me. Felipe da Costa Negrão - Universidade Federal do Amazonas

Prof $^{a}$ Dr $^{a}$ Germana Ponce de Leon Ramírez - Centro Universitário Adventista de São Paulo

Prof. Me. Gevair Campos - Instituto Mineiro de Agropecuária

Prof. Me. Givanildo de Oliveira Santos - Secretaria da Educação de Goiás

Prof. Dr. Guilherme Renato Gomes - Universidade Norte do ParanáProf. Me. Gustavo Krahl Universidade do Oeste de Santa Catarina

Prof. Me. Helton Rangel Coutinho Junior - Tribunal de Justiça do Estado do Rio de Janeiro

Prof $^{\mathrm{a}} \mathrm{Ma}$. Isabelle Cerqueira Sousa - Universidade de Fortaleza

Prof $^{a}$ Ma. Jaqueline Oliveira Rezende - Universidade Federal de Uberlândia

Prof. Me. Javier Antonio Albornoz - University of Miami and Miami Dade College

Prof. Me. Jhonatan da Silva Lima - Universidade Federal do Pará

Prof. Dr. José Carlos da Silva Mendes - Instituto de Psicologia Cognitiva, Desenvolvimento Humano e Social

Prof. Me. Jose Elyton Batista dos Santos - Universidade Federal de Sergipe

Prof. Me. José Luiz Leonardo de Araujo Pimenta - Instituto Nacional de Investigación Agropecuaria Uruguay

Prof. Me. José Messias Ribeiro Júnior - Instituto Federal de Educação Tecnológica de Pernambuco

Prof $^{\mathrm{a}} \mathrm{Dr}^{\mathrm{a}}$ Juliana Santana de Curcio - Universidade Federal de Goiás

Prof ${ }^{a}$ Ma. Juliana Thaisa Rodrigues Pacheco - Universidade Estadual de Ponta Grossa

Prof $^{a}$ Dr $^{a}$ Kamilly Souza do Vale - Núcleo de Pesquisas Fenomenológicas/UFPA

Prof. Dr. Kárpio Márcio de Siqueira - Universidade do Estado da Bahia

Prof $^{a}$ Dr $^{a}$ Karina de Araújo Dias - Prefeitura Municipal de Florianópolis

Prof. Dr. Lázaro Castro Silva Nascimento - Laboratório de Fenomenologia \& Subjetividade/UFPR

Prof. Me. Leonardo Tullio - Universidade Estadual de Ponta Grossa

Prof ${ }^{a}$ Ma. Lilian Coelho de Freitas - Instituto Federal do Pará

Prof $^{a}$ Ma. Liliani Aparecida Sereno Fontes de Medeiros - Consórcio CEDERJ

Prof $^{a}$ Dr $^{\mathrm{a}}$ Lívia do Carmo Silva - Universidade Federal de Goiás

Prof. Dr. Lucio Marques Vieira Souza - Secretaria de Estado da Educação, do Esporte e da Cultura de Sergipe

Prof. Me. Luis Henrique Almeida Castro - Universidade Federal da Grande Dourados

Prof. Dr. Luan Vinicius Bernardelli - Universidade Estadual do Paraná

Prof. Dr. Michel da Costa - Universidade Metropolitana de Santos

Prof. Dr. Marcelo Máximo Purificação - Fundação Integrada Municipal de Ensino Superior

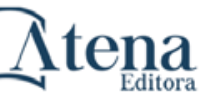

Ano 2020 
Prof. Me. Marcos Aurelio Alves e Silva - Instituto Federal de Educação, Ciência e Tecnologia de São Paulo

Prof ${ }^{a}$ Ma. Maria Elanny Damasceno Silva - Universidade Federal do Ceará

Prof ${ }^{a}$ Ma. Marileila Marques Toledo - Universidade Federal dos Vales do Jequitinhonha e Mucuri

Prof. Me. Ricardo Sérgio da Silva - Universidade Federal de Pernambuco

Prof ${ }^{a}$ Ma. Renata Luciane Polsaque Young Blood - UniSecal

Prof. Me. Robson Lucas Soares da Silva - Universidade Federal da Paraíba

Prof. Me. Sebastião André Barbosa Junior - Universidade Federal Rural de Pernambuco

Prof $^{a}$ Ma. Silene Ribeiro Miranda Barbosa - Consultoria Brasileira de Ensino, Pesquisa e Extensão

Prof $^{a}$ Ma. Solange Aparecida de Souza Monteiro - Instituto Federal de São Paulo

Prof. Me. Tallys Newton Fernandes de Matos - Faculdade Regional Jaguaribana

Prof $^{a}$ Ma. Thatianny Jasmine Castro Martins de Carvalho - Universidade Federal do Piauí

Prof. Me. Tiago Silvio Dedoné - Colégio ECEL Positivo

Prof. Dr. Welleson Feitosa Gazel - Universidade Paulista 
Editora Chefe: Prof $^{\mathrm{a}} \mathrm{Dr}^{\mathrm{a}}$ Antonella Carvalho de Oliveira

Bibliotecária: Janaina Ramos

Diagramação: Camila Alves de Cremo

Correção: Vanessa Mottin de Oliveira Batista

Edição de Arte: Luiza Alves Batista

Revisão: Os Autores

Organizadores: Henrique Ajuz Holzmann

João Dallamuta

Dados Internacionais de Catalogação na Publicação (CIP)

D451 O desenvolvimento sustentável na engenharia civil 2 /

Organizadores Henrique Ajuz Holzmann, João

Dallamuta. - Ponta Grossa - PR: Atena, 2020.

Formato: PDF

Requisitos de sistema: Adobe Acrobat Reader Modo de acesso: World Wide Web

Inclui bibliografia

ISBN 978-65-5706-614-0

DOI 10.22533/at.ed.140202511

1. Engenharia civil. 2. Desenvolvimento sustentável. I. Holzmann, Henrique Ajuz (Organizador). II. Dallamuta, João (Organizador). III. Título.

CDD 624

Elaborado por Bibliotecária Janaina Ramos - CRB-8/9166

Atena Editora

Ponta Grossa - Paraná - Brasil

Telefone: +55 (42) 3323-5493

www.atenaeditora.com.br

contato@atenaeditora.com.br 


\section{UTILIZAÇÃO DE BARREIRAS VERTICAIS NA REMEDIAÇÃO AMBIENTAL}

Data de aceite: 16/11/2020

Data de submissão: 07/09/2020

\section{Luciana Regina Cajaseiras de Gusmão}

Universidade Federal de Pernambuco,

Departamento de Engenharia Civil

Recife - Pernambuco

http://lattes.cnpq.br/4228865260590782

José Fernando Thomé Jucá

Universidade Federal de Pernambuco,

Departamento de Engenharia Civil

Recife - Pernambuco

http://lattes.cnpq.br/9551646929257730

Karla Salvagni Heineck

Universidade Federal do Rio Grande do Sul,

Departamento de Engenharia Civil

Porto Alegre - Rio Grande do Sul http://lattes.cnpq.br/2053839883513170

RESUMO: O presente trabalho traz explanação teórica compilada sobre os principais métodos de contenção de contaminantes utilizados na atualidade, as barreiras verticais, a fim de divulgar e disseminar as práticas. A contenção da área-fonte de contaminação é um método de custo relativamente baixo que consegue evitar o alastramento da pluma de contaminantes; seu princípio básico é controlar o movimento horizontal de água e contaminantes, dificultando o fluxo. As barreiras de solo-bentonita, cimentobentonita e solo-cimento-bentonita estão entre as mais frequentemente utilizadas. Em comparação, a primeira apresenta a vantagem de ser mais competitiva e proporcionar menores coeficientes de condutividade hidráulica, enquanto garante menor capacidade de suporte, está mais sujeita a misturas heterogêneas e necessita de maiores áreas de trabalho. Por outro lado, o cimento das cortinas de cimento-bentonita pode provocar gradativas trocas catiônicas que comprometam a estabilidade e permeabilidade da barreira. As cortinas de solo-cimento-bentonita buscam equilibrar baixa condutividade hidráulica e alta resistência.

PALAVRAS-CHAVE: Barreiras verticais, remediação ambiental, solo-bentonita, cimentobentonita, solo-cimento-bentonita.

\section{USE OF SLURRY WALLS IN ENVIRONMENTAL REMEDIATION}

ABSTRACT: The present work provides a compiled theoretical explanation about the main contaminant containment methods currently used, slurry walls, in order to disseminate and spread these practices. Containment of the contamination source area is a relatively low-cost method that manages to prevent the spread of the contaminant plume; its basic principle is to control the horizontal movement of water and contaminants, making the flow difficult. The soilbentonite, cement-bentonite and soil-cementbentonite barriers are among the most frequently used. In comparison, the former has the advantage of being more competitive and providing lower coefficients of hydraulic conductivity, while guaranteeing less carrying capacity, is more subject to heterogeneous mixtures and requires larger work areas. On the other hand, the cement of the cement-bentonite liners can cause gradual 
cationic exchanges that compromise the stability and permeability of the barrier. Soilcement-bentonite walls seek to balance low hydraulic conductivity and high strength.

KEYWORDS: Slurry walls, environmental remediation, soil-bentonite, cementbentonite, soil-cement-bentonite.

\section{I INTRODUÇÃO}

A total remediação de áreas contaminadas de forma a possibilitar uso irrestrito do solo e da água tem se mostrado ser um objetivo ilusório, já que os processos são bastante desafiadores e dispendiosos. As limitações das tecnologias de remediação, a frequente realização de uma investigação incompleta e definição imprecisa da problemática contaminante, além de natureza complexa e heterogênea da subsuperfície são as principais causas de insucesso na remediação (SMYTH et al., 2001).

A complexidade dos modelos de dispersão de contaminantes e a dificuldade associada da remediação crescem à medida que há mistura de fontes de contaminação, heterogeneidade da subsuperfície, configurações hidrogeológicas complicadas associadas com rochas fraturadas, ou mesmo interações entre diferentes contaminantes (SMYTH et al., 2001). É interessante notar que embora a massa de contaminantes na fonte geralmente exceda bastante a presente nas plumas (MACKAY; CHERRY, 1989), os contaminantes móveis são comumente os que geram mais riscos imediatos ao meio ambiente. Assim, as características das zonas de fonte e dispersão (plumas) são distintas, quase sempre requerendo tecnologias diferentes para remediação.

É importante que várias possibilidades sejam levadas em consideração para a escolha do método mais interessante para cada caso de remediação ambiental, inclusive quanto à possibilidade de combinar soluções, sempre tendo em mente o potencial de degradação e o custo, o qual envolve as condições de execução.

O presente trabalho apresenta explanação teórica compilada comparativa sobre os principais métodos de isolamento da zona-fonte, as cortinas verticais, com a finalidade de divulgar e disseminar essa prática cada vez mais.

\section{I REMEDIAÇÃO DE ÁREAS CONTAMINADAS}

A escolha e desenvolvimento de um programa de remediação para determinada área depende dos objetivos buscados em cada caso, podendo envolver a adoção de várias ações. Em alguns casos é possível fazer uso apenas de mecanismos físicos, químicos e biológicos naturais (atenuação natural) para reduzir a contaminação a níveis aceitáveis. No entanto, frequentemente é necessário fazer uso também de abordagens mais complexas, nas quais se deve almejar: a remoção/ 
destruição da massa contaminação na zona-fonte para evitar posterior propagação de pluma; o isolamento da zona-fonte através de barreiras hidráulicas ou físicas; ou controle e remediação da pluma através de sistema de bombeamento direto ou uso de barreiras reativas in situ (SMYTH et al., 2001).

Uma medida quase essencial quando não há capacidade tecnológica e financeira para restauração total da zona-fonte é o seu isolamento e contenção. A contenção da contaminação é obtida a partir da construção de cortinas/barreiras/ trincheiras verticais de baixa permeabilidade, contenções hidráulicas de longo-prazo com sistemas de bombeamento e tratamento dos contaminantes, ou intercepção e tratamento in situ da água subsuperficial contaminada através de barreiras reativas passivas (CHERRY; FEENSTRA; MACKAY, 1996).

Neste trabalho, serão abordados os principais tipos de contenções através de barreiras verticais de baixa condutividade hidráulica.

\subsection{Remediação por barreiras verticais}

A instalação de cortinas verticais geralmente se restringe a profundidades menores que $50 \mathrm{~m}$ e pode ter limitações em configurações geológicas difíceis, como terrenos rochosos com superfícies fraturadas (SMYTH et al., 2001). Utilizadas na Engenharia Civil para controle hidráulico em escavações desde a década de 50, apenas a partir da década de 70 as barreiras foram adaptadas para uso ambiental, difundindo-se nos anos 80 como componente de sistema de remediação de contaminantes (LEMOS, 2006). A contenção deve ser estrategicamente posicionada de forma a conter, divergir ou encapsular o fluxo.

Seu princípio básico é controlar o movimento horizontal de água e contaminantes, dificultando o fluxo de água e/ou o transporte advectivo de contaminantes através da cortina (SMYTH et al., 2001). Na definição dos objetivos da barreira, é essencial definir se ela vai atuar com baixa condutividade hidráulica, reduzindo o fluxo de água, ou impedindo o transporte de contaminantes, o qual se dá de forma mais complexa. Assim, a condutividade hidráulica é a principal preocupação desse tipo de projeto, assim como os parâmetros de resistência, custos e métodos executivos (LEMOS, 2006).

O projeto a ser elaborado depende dos objetivos da barreira. A maioria das intervenções de remediação com cortinas verticais, no entanto, utilizam técnicas combinadas para atingir seus objetivos de forma mais eficiente. Na geotecnia ambiental geralmente as barreiras verticais são dispostas em posicionamento circunferencial, ao redor da fonte de contaminação, frequentemente associadas a sistemas de drenagem subsuperficial e coberturas sobre a área contaminada para proteger a água subsuperficial off-site. No entanto, esse formato não é essencial, pois as barreiras podem ser dispostas de forma a isolar apenas parte do local. As 
barreiras também podem ser utilizadas para reduzir a migração de água limpa não contaminada para pontos de alto gradiente de concentração - pelo princípio da osmose (EVANS, 1993).

Segundo, Mitchell e Van Court (1997) e Devlin e Parker (1996), o potencial contaminante acumulado e a movimentação através da cortina a partir da difusão podem ser considerações importantes do projeto de isolamento.

A eficiência de um isolamento pode ser otimizada quando sua base é engastada em um aquitardo ou camada de baixa permeabilidade (SMYTH et al., 2001). O engaste se torna desnecessário quando há retirada da água contaminada ou os contaminantes estão concentrados próximo à superfície ou flutuando no nível freático (EVANS, 1993).

Onde essas condições geológicas não permitem ou o aquitardo é muito profundo, as cortinas podem ser limitadas dentro do aquífero, situação denominada por Cherry, Feenstra e Mackay (1996) de "cortina suspensa" (tradução nossa). Essa configuração é possível quando o gradiente for ascendente ou o contaminante for menos denso que a água, situações em que os contaminantes só são retidos se a barreira se estender em profundidade abaixo da base da área-fonte, e podem ser necessárias maiores taxas de bombeamento de água para manter a contenção hidráulica.

Recomenda-se maximizar o isolamento com o uso de sistema de cobertura acoplado às cortinas verticais. A cobertura tem função de reduzir a infiltração, controlar a eliminação de gases e vapores e garantir isolamento da subsuperfície contaminada com a biosfera (SMYTH et al., 2001). A maioria dos sistemas de contenção vertical necessita da proteção proporcionada pela cobertura, já que os materiais estão sujeitos a ressecamento e ciclos de gelo-degelo. O projeto final deve considerar o carregamento do tráfego (paralelo e transversal, se for o caso) e alternativas de integração com a paisagem (EVANS, 1993). É muito comum a utilização de geossintéticos (geogrelhas, geotêxteis ou geomembranas) ou argila compactada para essa finalidade.

Pode-se ainda acrescentar base às cortinas verticais para aumentar o isolamento dos contaminantes. Embora tenha havido evolução das tecnologias de execução de base nas barreiras, seus custos são elevados e a verificação de sua disposição e desempenho são difíceis, razão pela qual sua utilização não é muito comum no controle de remediação, sendo mais frequente em aterros sanitários (PETERSON; LANDIS, 1995).

Após a identificação dos objetivos pretendidos com a barreira, é necessário avaliar quais tipos de barreira atendem ao pretendido, geralmente a partir de modelos de fluxo de água e contaminantes. É necessário avaliar a hidrogeologia e aspectos geotécnicos do local, além de desempenho a curto e longo prazo de cada 
caso, especialmente do ponto de vista de condutividade hidráulica, resistência e compressibilidade (EVANS, 1993).

Interações físico-químicas entre materiais da cortina e contaminantes podem influenciar o desempenho hidráulico da contenção. Interações entre argila e componentes miscíveis na água como co-solventes ou surfactantes podem provocar retração de minerais argilosos, o que pode ocasionar aumento da condutividade hidráulica, efeito minimizado em profundidades com tensões confinantes maiores (SMYTH et al., 2001).

Materiais poliméricos também estão sujeitos a reações degradantes com fluidos orgânicos como hidrocarbonetos aromáticos e solventes de petróleo ou solventes de cloro e petróleo, tendo sua eficiência na aplicação de cortinas de contaminantes questionável, a depender do caso (MITCHELL; VAN COURT, 1997). A eficiência de uma barreira hidráulica vertical está diretamente ligada às propriedades do material de preenchimento utilizado, o qual depende especialmente da graduação do solo e percentual de bentonita utilizados para cortinas de solobentonita e solo-cimento-bentonita (LEMOS, 2006; PAGANI, 2007).

Os principais tipos de barreiras utilizadas são: trincheiras de solo-bentonita (com ou sem geomembrana), cimento-bentonita ou solo-cimento-bentonita, cortinas de concreto vibrado, colunas jet ground, estacas-prancha de aço convencional e paredes-diafragma (SMYTH et al., 2001). O desempenho de cada dependerá de características da contaminação e condições do projeto.

De forma geral, as barreiras de solo-bentonita estão entre as técnicas mais utilizadas devido à maior rapidez de execução e menor custo, seguidas das barreiras de cimento-bentonita ou solo-cimento-bentonita, de maior resistência (LEMOS, 2006). Tendo em vista que o custo é um critério decisivo na escolha da solução empregada, serão aprofundados esses três métodos.

\subsubsection{Barreiras de solo-bentonita}

Nesse método, trincheiras são escavadas e utiliza-se lama bentonítica (segundo Evans (1993), normalmente na proporção de 5\% de bentonita para 95\% de água, em peso) para manter a estabilidade geotécnica durante a escavação, a partir da pressão do fluido que excede o empuxo ativo do solo, e diminuir a condutividade hidráulica (BATISTA; LEITE, 2010). Para escavações até 20 m é possível utilizar retroescavadeira no processo, mas para profundidades maiores recomenda-se a utilização de dragline ou clamshell. O método de escavação utilizado não interfere diretamente na eficiência da barreira, mas é essencial que a camada impenetrável seja atingida continuamente (AZAMBUJA, 2004).

Segundo Azambuja (2004) e Lemos (2006), a escavação deve ser iniciada pela área de maior declividade do terreno. A largura da trincheira varia entre 0,6 e 1,5 
$\mathrm{m}$, sendo comum 0,9 m. Trincheiras muito estreitas são de difícil execução, enquanto que muito largas aumentam custos sem necessidade. Atingida a profundidade desejada, é feito reaterro na vala, quando parte da lama bentonítica é retirada e parte permanece recobrindo as paredes escavadas formando uma película fina (filter cake), a qual é a principal responsável pela redução da condutividade hidráulica da barreira (BATISTA; LEITE, 2010).

A lama consegue penetrar nos vazios do solo a partir de diferença de pressão existente, onde grupos de partículas sólidas, denominadas colóides, vão se acumulando até formação da do filter cake. Vale salientar que para evitar escorregamentos, as paredes devem estar na vertical durante a escavação, com um desvio máximo de $2 \%$ (XANTHAKOS, 1979).

Para garantia da estabilidade, a lama bentonítica deve permanecer próximo ao topo da trincheira (máximo de $90 \mathrm{~cm}$ abaixo) e pelo menos $60 \mathrm{~cm}$ acima do nível d'água (FILZ; HENRY; DAVIDSON, 1997). Caso contrário, a poropressão do solo adjacente exercerá uma força no filter cake que irá provocar sua desintegração, comprometendo, assim, o isolamento hidráulico e estabilidade do trecho, podendo ocorrer o colapso. Caso venha a ocorrer colapso, o trecho deve ser limpo antes da aplicação do material de preenchimento/reaterro (backfill) (XANTHAKOS, 1979).

O reaterro é feito a uma consistência de alto slump de concreto (100 - 150 $\mathrm{mm}$ ), de preferência com mistura de areia, silte, argila e lama bentonítica. Para atingir essa consistência, o material deve ser "fluidificado" a partir da adição da lama ao solo, e assim permanece no local ao longo do tempo (EVANS, 1993). É importante manter o peso específico da lama presente na vala sempre menor que o peso específico da mistura de reaterro solo-bentonita a fim de garantir a gradativa expulsão da bentonita a partir da inserção do backfill no trecho.

Adicionalmente, deve ser feita a retirada de eventuais sedimentos granulares existentes nas trincheiras antes da execução do reaterro para evitar a existência de pontos com maior condutividade hidráulica do que a mistura aplicada (D’APPOLONIA, 1980). O reaterro deve ser executado no extremo oposto do trecho em escavação, depositando-se continuamente a mistura solo-bentonita do fundo até a superfície, formando uma rampa com inclinação de 6:1 para que posteriormente escoe progressivamente sem ocorrência de segregação e expulsando a lama existente no trecho (LEMOS, 2006).

O slump adequado para cada caso deve ser determinado em estudos de laboratório para avaliar a condutividade hidráulica e compatibilidade com a execução. Ressalta-se que é importante que as amostras de ensaio sejam sempre preparadas com o mesmo slump do campo ou os dados não serão representativos da situação real. A compatibilidade química com os contaminantes envolvidos tem extrema importância, pois pode provocar aumento de permeabilidade da mistura 
(SMYTH et al., 2001).

É interessante notar que embora no estudo de Batista e Leite (2010) a adição de bentonita ao solo ensaiado não tenha gerado redução da condutividade hidráulica (na verdade, houve leve aumento), trouxe significativo crescimento da capacidade de retenção de contaminantes, aspecto muito positivo para os projetos de geotecnia ambiental.

O solo presente no backfill pode ser proveniente do próprio trecho escavado ou de empréstimo. Embora a utilização de solo local represente grande economia e frequentemente resulte em uma lama mais grossa/densa e com mais materiais finos em suspensão - o que promove redução da condutividade hidráulica (D'APPOLONIA, 1980), ressalta-se que é importante controlar a distribuição granulométrica do solo utilizado para resultar em uma mistura mais uniforme. Diante da variabilidade natural que pode haver ao longo da escavação, o controle deve ser mais fácil a partir da utilização de empréstimo (EVANS, 1993). Devido às altas profundidades que podem ser necessárias a depender do caso, é importante avaliar também a capacidade de suporte das misturas de reaterro (BATISTA; LEITE, 2010).

Vale ressaltar que a eficiência desse tipo de intervenção depende de condições da subsuperfície independentes de previsão e material de preenchimento utilizado (LEMOS, 2006), então as especificações técnicas de projeto só podem estabelecer garantias e responsabilização sobre aquilo que é possível controlar, como graduação do solo utilizado e quantidade de bentonita na mistura.

$\mathrm{Na}$ execução construtiva, pode-se optar por fazer a mistura de reaterro ao longo da trincheira ou em área remota de mistura. O primeiro método tem a vantagem de ser mais barato, já que não necessita de equipamentos mais especializados, e desvantagem de requerer maior área disponível para fazer a mistura e dificuldade de adicionar finos à mistura retirada na escavação. Assim, com a utilização de área remota de mistura é possível garantir maior homogeneidade, uniformidade e controle à mistura (EVANS, 1993).

A principal preocupação executiva se relaciona com a possibilidade de aumento da condutividade hidráulica, como no caso de mistura solo-bentonita mal executada, que pode gerar bolsões de solo ou mesmo de lama bentonítica, resultando em zonas de condutividade hidráulica maiores que o desejado. Ciclos de congelamento-descongelamento ou molhagem-secagem, ou mesmo o ressecamento da mistura solo-bentonita também podem ser fatores de risco da contenção (EVANS, 1993). Um projeto adequado de sistema de cobertura das cortinas pode prevenir o congelamento da estrutura.

Quanto ao comportamento de longo-prazo, a incompatibilidade química entra como principal consideração, sendo necessário controlar os aumentos de condutividade hidráulica. Pode-se minimizar o efeito do ataque químico com a 
utilização de solo bem-graduado com grande quantidade de finos, já que corresponde a um conjunto de grãos progressivamente menores relativamente inertes (EVANS, 1993). Vale salientar que os minerais argilosos possuem excesso de cargas elétricas em sua superfície e grande superfície específica, o que favorece fenômenos de interação elétrica e química.

\subsubsection{Barreiras de cimento-bentonita}

Também executada através de escavação, a principal diferença construtiva das trincheiras de solo-bentonita é que a lama utilizada na estabilização da escavação é comumente deixada para endurecer no local. Nesse caso, a lama contém bentonita, cimento e água, atingindo condutividade hidráulica na ordem de 1 $\times 10^{-5}$ e $1 \times 10^{-6} \mathrm{~cm} / \mathrm{s}$, enquanto que as de solo-bentonita resultam em valores entre $1 \times 10^{-7}$ e $1 \times 10^{-8} \mathrm{~cm} / \mathrm{s}$ (EVANS, 1993).

Como o solo escavado não é utilizado na mistura de reaterro, a destinação final desse material deve ser levada em consideração na análise das alternativas, já que envolve custos elevados de movimentação de terras e/ou bota-fora (EVANS, 1993). Em geral, esse método tem maior custo também devido à utilização de cimento (BATISTA; LEITE, 2010), entretanto, torna-se uma opção interessante quando não há na área solo adequado disponível para a mistura do backfill de solobentonita (PEARLMAN, 1999).

Esse tipo de barreira necessita de menos área de trabalho que a cortina de solo-bentonita, já que não realiza mistura com solo e o equipamento de mistura cimento-bentonita é bem compacto. O potencial para defeitos construtivos também é bem menor. A principal preocupação é a dosagem adequada de cimento, bentonita e água (EVANS, 1993). É recomendável quando a resistência é um requisito importante, já que o cimento proporciona aumento da capacidade de suporte com o tempo. Por outro lado, é uma barreira, em geral, mais permeável.

Millet e Perez (1981) apontam que o aumento de resistência possibilita execução de barreiras mais estreitas, geralmente variando entre 0,6 e 0,9 m para profundidades de até $30 \mathrm{~m}$. No caso de escavação por painéis alternados ou para pequenas profundidades costuma-se utilizar a própria lama de cimento-bentonita para manter a estabilidade do talude, deixando-a endurecer no local. Profundidades maiores que $30 \mathrm{~m}$ talvez não possibilitem manter a mistura com cimento trabalhável durante todo o tempo necessário, situação em que será necessário utilizar lama bentonítica durante a escavação para posterior substituição por outra com cimento ao término, a qual deve ser mais densa para possibilitar a expulsão (JEFFERIS, 1981).

O cimento interfere nas propriedades químicas da bentonita reduzindo sua capacidade de inchamento e retenção de água. Embora a condutividade hidráulica 
tenha pouca relação com a quantidade de cimento empregada, a mesma é função do tempo decorrido, já que o processo de hidratação do cimento proporciona redução da permeabilidade (LEMOS, 2006).

O contato entre o cimento e a lama bentonítica ocasiona floculação mútua, responsável pelo rápido enrijecimento da mistura. A agregação das partículas de argila provoca a progressiva quebra da estrutura em estado de gel da bentonita, retornando ao estado fluido após alguns minutos (JEFFERIS, 1981). A floculação que ocorre promove um filter cake mais poroso e permeável do que o da lama bentonítica apenas, permitindo maior escoamento por entre os poros do solo. Assim, além de criar barreiras mais permeáveis, há perda considerável de lama para o solo (pode ser necessário utilizar o dobro do volume teórico de material) (XANTHAKOS, 1979).

Para que as juntas frias de execução durante o preenchimento interrompido não se tornem pontos fracos do sistema (com maior condutividade hidráulica), é necessário que seja feita a cuidadosa limpeza de toda a superfície, garantindo aderência entre as camadas bombeadas (EVANS, 1993).

A mistura é preparada em misturador coloidal de alta velocidade, inicialmente com a mistura bentonita-água e posteriormente com adição do cimento para ser bombeada na vala (EVANS, 1993). Jefferis (1981) chama a atenção que para evitar a ocorrência do fenômeno chamado de "bleeding", em que há segregação bastante considerável dos materiais, a lama bentonítica deve ser completamente hidratada antes da adição do cimento (por pelo menos quatro horas). Quanto maior a proporção de bentonita em relação ao cimento, menor será a segregação, já que a bentonita é a responsável por suportar as partículas do cimento. Adicionalmente, Li et al. (1989) afirmam que a segregação ocorre mais rapidamente a depender de quanto menor for o fator água-cimento da mistura.

Alterações de propriedades ao longo do tempo são mais difíceis de ocorrer devido à hidratação do cimento e progressivo ganho de resistência com o tempo. No entanto, a possibilidade de incompatibilidade química, tanto global quanto local, deve ser considerada. Usualmente utiliza-se bentonita sódica na mistura, pois a cálcica produz lama mais instável e forma película mais permeável, por ser menos expansiva quando hidratada. No entanto, é possível que haja gradativa troca catiônica do sódio $\left(\mathrm{Na}^{+}\right)$da bentonita com as grandes quantidades de íons de cálcio $\left(\mathrm{Ca}^{2+}\right)$ liberados pelo cimento, transformando a bentonita sódica em cálcica ao longo do tempo, o que implica maior permeabilidade e instabilidade (BATISTA; LEITE, 2010).

\subsubsection{Barreiras de solo-cimento-bentonita}

O aumento do custo proveniente do acréscimo de cimento e maiores volumes 
de movimentação de terra, atrelado à maior permeabilidade e instabilidade química das cortinas de cimento-bentonita têm sido os principais aspectos motivacionais da utilização das barreiras de solo-cimento-bentonita, de desenvolvimento mais recente. A incorporação de solo na mistura provoca redução dos custos e maior estabilidade química, atrelada à maior resistência propiciada pelo cimento. É uma opção interessante em casos onde é possível utilizar o solo escavado na composição da mistura de preenchimento, reduzindo o volume de bota-fora (PEARLMAN, 1999).

Dessa forma, as barreiras de solo-cimento-bentonita tentam combinar a baixa condutividade hidráulica das barreiras de solo-bentonita com a maior resistência das barreiras de cimento-bentonita. Seu método construtivo é semelhante às barreiras tradicionais de solo-bentonita, porém geralmente com menores profundidades (até 15 m) (RYAN; DAY, 2002).

Assim como nas barreiras de solo-bentonita, a adição de finos pode melhorar a eficiência da contenção hidráulica, enquanto o excesso pode exigir maiores quantidades de água para atingir a trabalhabilidade necessária, reduzindo a densidade da mistura e, consequentemente, aumentando sua permeabilidade (PAGANI, 2007).

A condutividade hidráulica do material de preenchimento utilizado neste tipo de cortina é resultado de complexas interações entre os componentes da mistura. $\mathrm{O}$ cimento, além de alterar as propriedades bentoníticas mencionadas anteriormente, demanda mais água à mistura para que seja possível executar seu lançamento na vala, reduzindo, assim, a densidade do material de preenchimento - e aumentando a permeabilidade. É possível ainda fazer uso de aditivos, especialmente os retardadores de pega, na tentativa de reduzir a condutividade hidráulica, porém além de dificultar o processo executivo, aumenta os custos (LEMOS, 2006).

A avaliação de cada método deve levar em consideração também outros aspectos essenciais como resistência e durabilidade do backfill. Stavridakis (2005) mostrou que para misturas de solo granular, cimento (4\%) e bentonita, houve tendência à redução da resistência à compressão não confinada com o aumento percentual de bentonita, sendo a maior resistência obtida com $5 \%$ de bentonita, muito superior àquelas referentes às demais porcentagens estudadas $(10,15,20$, 25 e $30 \%$ ).

A resistência das barreiras é função do fator água-cimento e do tempo (de cura) (PAGANI, 2007). Segundo Azambuja (2004), quanto menor for o fator águacimento da mistura solo-cimento-bentonita, maior a resistência à compressão não confinada e resistência de pico (ao cisalhamento), e menor a condutividade hidráulica. É importante ressaltar, no entanto, que o acréscimo do cimento provoca aumento do custo da contenção quase em proporção direta com a resistência; e que seu excesso pode aumentar as chances de fissuração da barreira devido ao 
aumento de rigidez frente aos carregamentos sofridos (PAGANI, 2007).

Batista e Leite (2010), estudando misturas de solo-bentonita e solocimento-bentonita, constataram que a adição de cimento, embora tenha aumentado significativamente a resistência, também trouxe grandes aumentos de condutividade hidráulica, mesmo para pequenas porcentagens de cimento. Diante do objetivo principal das cortinas de conter a contaminação, o aumento de permeabilidade ocasionado deve ser bem estudado em cada caso, já que pode vir a comprometer a funcionalidade da obra.

Adicionalmente, Pagani (2007) verificou a viabilidade de adicionar fibras de polipropileno em barreiras de solo-cimento-bentonita. A adição proporcionou aumento significativo na resistência não-confinada, pequena redução de deformação na aplicação de cargas isotrópicas e redução a fragilidade da mistura, sem, entretanto, causar alterações na condutividade hidráulica. A adição em misturas de solo-bentonita também se mostrou viável, sem alterar significativamente o comportamento hidráulico da mistura.

\section{I CONSIDERAÇÕES FINAIS}

A utilização de cortinas de contenção são opções de remediação ambiental com custo relativamente baixo que conseguem impedir o alastramento da contaminação. É possível utilizar essa técnica em conjunto com meios de tratamento dos contaminantes a fim de obter uma remediação mais rápida da área.

A cortina de solo-bentonita apresenta a vantagem de ser mais competitiva e proporcionar menores coeficientes de condutividade hidráulica, enquanto garante menor capacidade de suporte, está mais sujeita a misturas heterogêneas e necessita de maiores áreas de trabalho. Por outro lado, embora a utilização de contenções de cimento-bentonita promova estruturas mais resistentes, o isolamento da área pode ficar comprometido a partir do aumento da condutividade hidráulica ao longo do tempo (devido à instabilidade química do sistema). Mais recentemente, tem crescido a utilização das barreiras de solo-cimento-bentonita, na tentativa de equilibrar a baixa condutividade hidráulica promovida pelas barreiras de solo-bentonita, com a maior resistência das de cimento-bentonita.

Vale salientar que é de extrema importância considerar a compatibilidade química da contenção com o meio e os contaminantes existentes, de forma que não comprometa a funcionalidade do bloqueio. Cada caso de remediação deve ser analisado detalhadamente para escolha do(s) método(s) que melhor atende(m) às necessidades. 


\section{REFERÊNCIAS}

AZAMBUJA, R. M. B. Comportamento mecânico e hidráulico de misturas de solocimento-bentonita para aplicação em barreiras verticais de contenção de contaminantes. Dissertação (Mestrado em Engenharia Civil): Universidade Federal do Rio Grande do Sul, 2004. 99p.

BATISTA, P.; LEITE, A. do L. Misturas de um solo laterítico com cimento e bentonita para uso em cortinas verticais. R. Esc. Minas. Ouro Preto, v. 63, n. 2, p. 255-263, abr./jun. 2010.

CHERRY, J. A.; FEENSTRA, S.; MACKAY, D. M. Concepts for remediation of sites contaminated with DNAPLS. In: PONKOW, J. F; CHERRY, J. A. (Eds.). Dense chlorinated solvents and other DNAPLs in groundwater. Ontario, Canada: Waterloo Press, 1996. p. 475-506.

D'APPOLONIA, D. J. Soil-bentonite slurry trench cutoff. Journal of the Geotechnical Engineering Division. New York, v. 107, n. 4, p. 393-409, 1980.

DEVLIN, J. F.; PARKER, B. L. Optimun hydraulic condutivity to limit contaminant flux through cutoff walls. Ground Water. v. 34, n. 4, p. 719-726, 1996.

EVANS, J. C. Vertical cutoff walls. In: DANIEL, D. E. (Ed.). Geotechnical practice for waste disposal. London, England: Chapman \& Hall, 1993. p. 430-454.

JEFFERIS, S.A. Bentonite-cement slurries for hydraulic cut-offs. International Conference Soil Mechanics and Foundation Engineering. Stockholm, v. 1, p. 435-440, Jun/1981.

FILZ, G. M.; HENRY, L. B.; DAVIDSON, R. R. Formation and properties of bentonita filter cakes. In: REDDI, L. N.; BONALA, M. S. Filtration and drainage in geotechnical/geoenvironmental engineering (Geotech. Spec. Publ. n. 78). New York: ASCE, 1998. p. 69-88.

LEMOS, R. G. Estudo do comportamento hidráulico, mecânico e químico de barreiras hidráulicas verticais, compostas por solo residual, bentonita e cimento sob ação de substâncias agressivas. 2006. Tese (Doutorado em Engenharia Civil): Universidade Federal do Rio Grande do Sul, 2006. 310p.

LI, J. C.; HWANG, C. L.; YAO, H. L.; LEE, H. J.; LEE, R. J. A study of slag cement-bentonite slurry, In: International Conference on Soil Mechanics and Foundation Engineering, $12^{\mathrm{a}}$ edição (Rio de Janeiro). Rotterdam: A. A. Balkema, v. 3, p. 1499-1502, 1989.

MACKAY, D. M.; CHERRY, J. A. Groundwater contamination: limits of pump-and-treat remediation. Environmental Science \& Technology, v. 23, n. 6, p. 630-636, 1989.

MILLET, R. A.; PEREZ, J. Current USA practice: slurry wall specification. Journal of the Geotechnical Engineering Division. ASCE, v. 107, n. 8, p. 1041-1052, 1981.

MITCHELL, J. K.; VAN COURT, W. A. N. Barrier design and installation: walls and covers. In: WARD, C. H.; CHERRY, J. A.; SCALF, M. R. (Eds.). Subsurface restoration. Michigan, EUA: Ann Arbor Press, 1997. p. 175-197. 
PAGANI, B. R. Estudo de misturas solo-cimento-bentonita-fibra para uso em barreiras verticais de contenção de contaminantes. Dissertação (Mestrado em Engenharia Civil): Universidade Federal do Rio Grande do Sul, 2007. 116p.

PEARLMAN, L. Subsurface containment and monitoring systems: barriers and beyond (overview report). Washington: National Network of Environmental Management Studies Fellow, 1999. 61p.

PETERSON, M. E.; LANDIS, R. C. Artificially emplaced floors and bottom barriers. In: RUMER, R. R.; MITCHELL, J. K. (Eds.). Assessment of barrier containment technologies: a comprehensive treatment for environmental remediation applications. US Department of Energy/US Environmental Protection Agency: Dupont Company, 1995. p. 185-209.

RYAN, C. R.; DAY, S. R. Soil-cement-bentonite slurry walls. In: O'NEILL, M. W.; TOWNSEND, F. C. Deep Foundations 2002: An international perspective on theory, design, construction and performance (Geotechnical special publication n. 16). New York: ASCE, 2002. p. 713-727.

SMYTH, D. J. A.; GILHAM, R. W.; BLOWES, D. W.; CHERRY, J. A. In situ containment and treatment of contaminated soil and groundwater. In: ROWE, R. K. (Ed.). Geotechnical and geoenvironmental engineering handbook. Ontario, Canada: Kluwer Academic Publishers, 2001. p. 921-945.

STAVRIDAKIS, E. Presentation and assessment of clay influence on engineering parameters of cement-treated clayed mixtures. EJGE, v. 10, bundle A, 2005.

XANTHAKOS, P. P. Slurry Walls. New York: McGraw Hill, 1979. 622p. 